\title{
First Note on the Definition of $s_{2}$-Convexity
}

\author{
I. M. R. Pinheiro \\ A'beckett st, Melbourne, Victoria, Australia \\ E-mail: illmrpinheiro@gmail.com \\ Received January 7, 2011; revised January 18, 2011; accepted January 25, 2011
}

\begin{abstract}
In this short, but fundamental, note, we start progressing towards a mathematically sound definition of the real functional classes $K_{s}^{2}$.
\end{abstract}

Keywords: Convex, $S$-convex, s-convex, $s_{2}$-convex, $S_{2}$-Convex, Real Function

\section{Introduction}

As seen in [1], the boundaries of each one of the functional classes $K_{s}^{2}$ are currently determined through the following definition:

Definition 1. A function $f: X->\Re$ is said to be $s_{2}$-convex if the inequality

$$
f(\lambda x+(1-\lambda) y) \leq \lambda^{s} f(x)+(1-\lambda)^{s} f(y)
$$

holds $\forall \lambda \in[0,1] ; \forall x, y \in X ; s: 0<s \leq 1 ; X \subseteq \mathfrak{R}_{+}$.

Remarks:

- If the complementary inequality to the above inequality is verified then $f$ is told to be $\mathrm{s}_{2}$-concave;

- To recover the definition of real convex functions, it suffices making $s=1$ in the above inequality, deleting the symbol ' $s_{2}$ ' ' in the definition, and replacing $\mathfrak{R}_{+}$with $\mathfrak{R}$ in the definition.

Unfortunately, a few real convex functions have been left out of the just mentioned functional classes because of the wording of the current definition of such classes.

It is just that each one of the functional classes $K_{s}^{2}$ is supposed to contain, in itself, the entire class formed by the real convex functions!

We are then facing an impossible-to-deny-urgency of fixing the above mentioned definition.

The titles of the sections of this paper are:

1. Introduction;

2. Counter-examples;

3. Proposed fixing;

4. Conclusions;

5. References.

\section{Counter-Examples}

The easiest way to produce evidence to the claim that the current definition of the classes $K_{s}^{2}$ is equivocated is to present a convex function that does not belong to $K_{s}^{2}$ for some allowed value of $s$. We here will actually exhibit a model from which to generate such convex functions, so that we can choose one of them to present.

Consider a real convex function (any) that is negative in its entire domain.

Take its domain to be the real interval [a, b] and the function to be $f$.

Because $f$ is convex, it is definitely true that, for each $\{x, y\} \subset D_{f}$, we have:

$$
\begin{gathered}
f(\lambda x+(1-\lambda) y) \leq \lambda f(x)+(1-\lambda) f(y), \\
\forall \lambda \in[0,1] .
\end{gathered}
$$

Assume that $f$ is also $s_{2}$-convex, for all allowed values of $s_{2}$, as it would have to be the case in order for us to be entitled to state that $K_{s}^{2}$ extends the class of convex functions.

After considering the just mentioned assumption, we will then have that

$$
\begin{gathered}
f(\lambda x+(1-\lambda) y) \leq \lambda^{\mathrm{s}} f(x)+(1-\lambda)^{\mathrm{s}} f(y), \\
\forall \lambda \in[0,1] .
\end{gathered}
$$

Because $f(x) \neq|f(x)|$, it is true that $\lambda^{s} f(x) \leq \lambda f(x)$ and it is also true that $(1-\lambda)^{s} f(y) \leq(1-\lambda) f(y)$. Consequently, it is true that

$$
f(\lambda x+(1-\lambda) y) \leq \lambda^{s} f(x)+(1-\lambda)^{s} f(y) \leq \lambda f(x)+(1-\lambda) f(y) .
$$


However, the last inequality frontally contradicts the assumption that $K_{s}^{2}$ extends $K_{1}^{2}$, that is, that $K_{s}^{2}$ extends the class of convex functions!

Therefore, it can only be the case that all the fully negative real convex functions have been left out of the current definition of $s_{2}$-convexity, and at least all of those have been left out of the current definition, which, therefore, cannot be a mathematically acceptable definition at least in terms of its analytical description.

To exemplify the just mentioned missing convex functions, we consider the function $f(x)=x^{2}-5$ when $x \in[0,2] . f$ is clearly a convex function, due to its graph and the geometric definition of convex functions, which does match their analytical definition. In this case, $f$ should also be $s_{2}$-convex, as explained before. However, see:

$$
\begin{gathered}
f(\lambda x+(1-\lambda) y) \leq \lambda^{\mathrm{s}} f(x)+(1-\lambda)^{\mathrm{s}} f(y) \\
\Leftrightarrow(\lambda x+(1-\lambda) y)^{2}-5 \leq \lambda^{\mathrm{s}}\left(x^{2}-5\right)+(1-\lambda)^{\mathrm{s}}\left(y^{2}-5\right) .
\end{gathered}
$$

The last step is clearly untrue for at least $x=0, y=1$, $\lambda=0.5$, and $s=0.5$.

\section{Proposed Fixing}

We propose two definitions to replace the current definition of the classes $K_{s}^{2}$.

Our definitions should keep the intended geometry of the functions that are supposed to extend the convex functions and, at the same time, provide us with a more mathematically acceptable analytical interpretation of the phenomenon $s_{2}$-convexity.

The proposed definitions are:

Definition 2. A function $f: X->\mathfrak{R}$, for which $|f(x)|=f(x)$, is told to belong to $K_{s}^{2}$, for some allowed and fixed value of $s$, if the inequality

$$
f(\lambda x+(1-\lambda) y) \leq \lambda^{s} f(x)+(1-\lambda)^{s} f(y)
$$

holds $\forall \lambda \in[0,1] ; \forall x, y \in X ; s: 0<s \leq 1 ; X \subseteq \Re_{+}$.

Definition 3. A function $f: X->\mathfrak{R}$, for which $|f(x)|=-f(x)$, is told to belong to $K_{s}^{2}$, for some allowed and fixed value of $s$, if the inequality

$$
f(\lambda x+(1-\lambda) y) \leq \lambda^{\frac{1}{s}} f(x)+(1-\lambda)^{\frac{1}{s}} f(y)
$$

holds $\forall \lambda \in[0,1] ; \forall x, y \in X ; s: 0<s \leq 1 ; X \subseteq \mathfrak{R}_{+}$.

Remark:

If the complementary inequality to one of the inequalities above is verified for some function $f$ then such a function is told to be $s_{2}$-concave.

\section{Conclusions}

In this short note, we have started to fix the analytical definition of the phenomenon $s_{2}$-convexity, which has been created with the intention of extending the phenomenon convexity, once if the geometric idea of the phenomenon has ever been correct then something went really wrong when transferring that idea to the 'Real Analysis world'.

We have exhibited an entire set of convex functions that had been left out of the classes $K_{s}^{2}$ because of the inappropriate wording of its current analytical definition. We then progressed to present a member of that set, the set of the 'forgotten convex functions', and, finally, to re-word the analytical definition of $s_{2}$-convexity so that the just mentioned set, formed by certain convex functions, were finally included in the classes that extend it.

\section{References}

[1] M. R. Pinheiro, “Convexity Secrets,” Trafford Publishing, Canada, 2008. ISBN: 1425138217. 